Meta

Journal des traducteurs

Translators' Journal

\title{
Hacia un programa curricular comun de traducción para America Latina
}

\section{Ileana Cabrera et Patricia Hörmann}

Volume 35, numéro 3, septembre 1990

La traduction dans le monde hispanolusophone

URI : https://id.erudit.org/iderudit/002185ar

DOI : https://doi.org/10.7202/002185ar

Aller au sommaire du numéro

\section{Éditeur(s)}

Les Presses de l'Université de Montréal

\section{ISSN}

0026-0452 (imprimé)

1492-1421 (numérique)

Découvrir la revue

Citer cet article

Cabrera, I. \& Hörmann, P. (1990). Hacia un programa curricular comun de traducción para America Latina. Meta, 35(3), 552-560.

https://doi.org/10.7202/002185ar
Résumé de l'article

Hoy más que nunca resulta especialmente urgente comprender a cabalidad el papel fundamental que la actividad traductora desempeña en el desarrollo y en el destino de una nación, o de toda una civilización. Hay dos propósitos que, para los efectos del presente trabajo, es menester recalcar : el primero de ellos dice relación con la traducción y el proceso de toma de decisiones y el segundo, con la traducción y la importancia del español como lengua de influencia.

Para neutralizar los efectos adversos producidos por las barreras lingüísticas, creemos necesario destacar aquellas estrategias que resulten más útiles para acceder a la información técnica, científica y cultural de nuestra era y que consisten en la formación de traductores a nivel universitario versus la enseñanza masiva de lenguas extranjeras.

En vista de la similitud de situaciones y condiciones en el mercado de la traducción en América Latina, postulamos la necesidad de Programas de Traducción en la enseñanza superior, con elementos comunes que permitan la formación de traductores capaces de satisfacer las exigencias del mercado ocupacional. Ahora más que nunca, necesitamos formar traductores que posean una metodología disciplinada y un conocimiento profundo de las materias que deban traducir en el ejercicio de su labor profesional, con el fin de garantizar una calidad uniformemente alta para los productos de su trabajo. Los estándares de rigor y análisis, la perspectiva interdisciplinaria, la vocación de compromiso permanente con la investigación y, sobre todo, el hábito de trabajo solidario y de crítica intersubjetiva son características de un modo de ser netamente universitario.
Tous droits réservés @ Les Presses de l'Université de Montréal, 1990
Ce document est protégé par la loi sur le droit d'auteur. L’utilisation des services d'Érudit (y compris la reproduction) est assujettie à sa politique d'utilisation que vous pouvez consulter en ligne.

https://apropos.erudit.org/fr/usagers/politique-dutilisation/ 


\title{
HACIA UN PROGRAMA CURRICULAR COMUN DE TRADUCCION PARA AMERICA LATINA
}

\author{
Ileana Cabrera y Patricia HöRmann \\ Pontificia Universidad Católica de Chile, Santiago, Chile
}

\section{RESUMEN}

Hoy más que nunca resulta especialmente urgente comprender a cabalidad el papel fundamental que la actividad traductora desempeña en el desarrollo y en el destino de una nación, o de toda una civilización. Hay dos propósitos que, para los efectos del presente trabajo, es menester recalcar: el primero de ellos dice relación con la traducción y el proceso de toma de decisiones y el segundo, con la traducción y la importancia del español como lengua de influencia.

Para neutralizar los efectos adversos producidos por las barreras linguísticas, creemos necesario destacar aquellas estrategias que resulten más útiles para acceder a la información técnica, científica y cultural de nuestra era y que consisten en la formación de traductores a nivel universitario versus la enseñanza masiva de lenguas extranjeras.

En vista de la similitud de situaciones y condiciones en el mercado de la traducción en América Latina, postulàmos la necesidad de Programas de Traducción en la enseñanza superior, con elementos comunes que permitan la formación de traductores capaces de satisfacer las exigencias del mercado ocupacional. Ahora más que nunca, necesitamos formar traductores que posean una metodología disciplinada y un conocimiento profundo de las materias que deban traducir en el ejercicio de su labor profesional, con el fin de garantizar una calidad uniformemente alta para los productos de su trabajo. Los estándares de rigor y análisis, la perspectiva interdisciplinaria, la vocación de compromiso permanente con la investigación $\mathrm{y}$, sobre todo, el hábito de trabajo solidario y de crítica intersubjetiva son características de un modo de ser netamente universitario.

En América Latina, estamos insertos en una realidad bien específica, que se caracteriza por un aumento creciente de la actividad de traducción, producto de un crecimento vertiginoso de las comunicaciones científicas y técnicas ( CST» Rondeau, 1984: 13) provenientes de países industrializados. Cualesquiera sea el país en el cual el traductor ha sido formado y, las características particulares de cada una de nuestras naciones, el traductor se verá enfrentado a situaciones como las siguientes que, por lo general, en nuestros países, tienden a repetirse:

- mayor volumen de demanda de traducciones técnico-científicas con respecto al volumen detraducciones literarias;

- similitud de idiomas extranjeros en que llega la información, entre los cuales predominan abiertamente el alemán, el francés y el inglés;

- carencia de especialistas y de herramientas que apoyen su labor profesional (documentalistas, terminólogos, revisores, terminológicos, etc.);

- necesidad de traducir textos a los idiomas extranjeros antes mencionados y

- falta de criterios comunes respecto a los derechos y obligaciones de un traductor.

Esta similitud de características, que dicen relación principalmente con la demanda de traducciones del mercado, hace pensar en la necesidad y conveniencia de que, a nivel 
latinoamericano, exista una formación superior con elementos comunes que satisfagan, en la forma más eficaz posible, los requerimientos planteados.

\section{ANTECENTES}

La actividad traductora ha existido desde la antiguiedad como un medio óptimo para transmitir y compartir el conocimiento necesario para la supervivencia y el desarrollo físico y espiritual de los hombres. En particular, en América Latina, su importancia radica en el hecho de que nuestros países están insertos en una realidad bien específica, cual es la de ser receptores de información proveniente, en su mayor parte, de naciones industrializadas.

Diversos autores señalan con acierto cómo las épocas de mayor esplandor y lucidez de la humanidad coinciden con episodios de intensa actividad en el área de las traducciones o son precedidas por un gran incremento de esta actividad. En todas las civilizaciones, las traducciones han permitido asegurar que los logros individuales, o de grupos muy pequeños, puedan conservarse y que los miembros de comunidades distanciadas cronológica, geográfica o culturamente puedan reconocerse como agentes solidarios de un quehacer compartido, cual es el enriquecimiento y el desarrollo de ese patrimonio de cultura y civilización, recogido por la especie humana en el curso de su historia. Tal patrimonio debe, naturalemente, ser puesto al alcance de todos, ya que a todos pertenece y debe estar destinado a contribuir al bien de toda la comunidad.

Hoy más que nunca resulta especialemente urgente comprender a cabalidad el papel fundamental que la actividad traductora desempeña en el desarrollo y destino de una nación, o de toda una civilización. Dentro de esta perspectiva y, considerando el tema central de nuestro trabajo, queremos destacar dos aspectos: el primero de ellos dice relación con la traducción y el proceso de toma de decisiones y el segundo, con la traducción y la importancia del español como lengua de influencia en el mundo contemporáneo.

En lo que se refiere al primer aspecto, en este momento histórico de relaciones mundiales tan complejas y de creciente interdependencia, es fundamental que aquellos que participan en la toma de decisiones estén informados oportuna y adecuadamente. Dirigentes y dirigidos, en todos los ámbitos de la actividad social, tanto nacional como internacional, se ven obligados a colaborar desde un suelo común de información compartida que haga posible y eficaz su interacción. Sin embargo, informarse requiere una inversión sustancial de recursos humanos y económicos, pues el caudal de información no solo es enorme, sino también de un alto grado de especialización. Además, porque este proceso no es un fenómeno de distribución homogénea. En otras palabras, la información se produce y se difunde aceleradamente en los países desarrollados, mientras que en aquellos en vías de desarrollo o no se produce o su difusión es tan retardada que sus efectos pueden llegar a ser imperceptibles. En general, es posible afirmar que los países que generan información y aquellos que contribuyen en este proceso elaboran decisiones más adecuadas que los pueblos receptores. Estos últimos suelen transformarse en grupos indiferentes e indefensos, sometidos a las inexorables consecuencias de las decisiones ajenas. Todo lo anterior nos lleva a concluir que los traductores y las traducciones son parte importante de las condiciones que permiten a un pueblo jugar, en este escenario mundial, el primero de los papeles, y una sociedad alerta debe advertirlo así. El permitir que las traducciones cumplan con la función de llevar conocimientos, ideas y experiencias, expresados en una lengua a otra lengua que así lo requiere, es una responsabilidad clara para los dirigentes de toda sociedad moderna.

En la era postindustrial, el liderazgo que ejercen los países ya no depende tanto del tamaño y ubicación de sus territorios, ni de los recursos naturales a su disposición - factores decisivos en otras épocas - sino más bien de su capacidad para generar y utilizar 
eficiente y oportunamente el bien más codiciado y rentable de nuestros tiempos, cual es la información. Bajo nuestro prisma, traducción es sinónimo de información y esta última es sinónimo de poder.

En segundo lugar, debemos destacar y recalcar el papel de la actividad traductora y su relación con la vigencia y esfera de influencia que tiene o pudiese tener el mundo hispanohablante y el papel que le cabría desempeñar dentro de la comunidad de naciones.

Las lenguas son siempre funcionales a la supervivencia y al desarrollo de los pueblos que las hablan y es por eso que determinadas lenguas adquieren mayor influencia cuando aseguran a los pueblos los bienes y las relaciones necesarias para su desarrollo.

Las lenguas dominantes en la actualidad, entre ellas, el alemán, el francés y el inglés, son los vehículos que diseminan el conocimiento científico y tecnológico que se produce hoy en día. Otras, como el japonés, el ruso, el chino, el italiano y el portugués, si bien pertenecen a países desarrollados, se mantienen en un segundo plano en relación con otras lenguas de mayor universalidad. Tanto es así que gran parte de las publicaciones, producto de investigaciones japonesas dirigidas al mundo occidental no se publican en esa lengua, ya que, o son comunicadas por los propios japoneses en inglés o son traducidas de immediato a otros idiomas. Lo mismo ocurre con los trabajos en ruso, immediatamente traducidos a otras lenguas y así difundidos hacia el resto del mundo.

Por ello resulta especialmente pertinente e importante mostrar la analogía de nuestra situación con los casos aludidos, pues si hoy, desde una perspectiva exclusivamente linguiística, es frágil la situación para las lenguas de aquellos pueblos como el japonés y el ruso que sí producen ciencia y tecnología de alta calidad e importancia, cuánto más precaria puede ser, a corto plazo, la supervivencia de las lenguas de aquellos pueblos, como los casi trescientos millones de hispanohablantes, que son, en alto grado, dependientes en los ámbitos de la creación científica y tecnológica.

Dos factores agravan la situación para los hispanohablantes: el poco tiempo del que disponen para cubrir la brecha que los separa de los pueblos generadores de información y así poder resolver su dependencia, y la escasez de medias que los obliga imperivamente a evitar toda duplicación de esfuerzos y recursos.

Más allá de estas razones, se hace necesario promover la actividad traductora para asegurar la supervivencia y el desarrollo de nuestra cultura y de nuestra lengua. Al hacerlo, protegemos la identidad que nos es propia, conservando el potencial de influencia necesario para contribuir al enriquecimiento de la comunidad internacional.

Frente a la necesidad de superar las barreras lingüísticas que podrían llevar consigo, a corto plazo, a una dependencia científica, política, económica y por qué no hasta cultural, todos los estados modernos y especialmente aquellos cuyas lenguas se encuentran en situación desmedrada, han diseñado dos estrategias diferentes con idéntico objectivo que es el de neutralizar estas barreras lingüísticas.

La primera de estas estrategias consiste en la enseñanza masiva de aquellas lenguas extranjeras que resulten más rentables para acceder a la información; la segunda, también muy importante, es la formación de traductores universitarios calificados. Suecia y los Países Bajos, entre otros, ilustran la aplicación de la primera de estas estrategias. El éxito alcanzado en ambos países, que se manifiesta en poblaciones prácticamente bilingües ha puesto, no obstante, en peligro el desarrollo del sueco y del holandés como lenguas de influencia internacional.

A nivel mundial, también ha sido desarrollada la segunda de las estrategias; prueba de ello es el número creciente de escuelas e institutos universitarios dedicados a la enseñanza de la traducción y a la investigación de su problemática. Tal es el caso de centros universitarios como los de Paris, Madrid, Leipzig, Heidelberg, Georgetown, 
Montréal, Trois-Rivières, Laval, McGill, Ottawa, por nombrar tan solo algunos, en los cuales el estudio de la traducción ocupa un sitio destacado.

La necesidad de establecer estudios académicos a nivel superior en América Latina se hizo sentir ya en las primeras décadas del presente siglo y, a partir de los años 40 , comienzan a crearse carreras de traducción en los diferentes países. Cabe mencionar a Argentina, en forma especial, ya que en este país se creó la primera carrera universitaria, la de Traductor Público, en 1945, en la Facultad de Ciencias Económicas de la Universidad Nacional de Buenos Aires, de donde pasó más tarde a la Facultad de Derecho. Le siguen Uruguay que también creó la carrera de Traductor Público años más tarde, en 1954, la que se imparte en la Facultad de Derecho de la Universidad Nacional. México inició estos estudios en 1966, a través del Instituto de Intérpretes y Traductores (IIT) el cual otorgó, en un principio, los títulos de Técnico-Traductor y Técnico Traductor-Intérprete y luego rápidamente desarrolló la Carrera de Traductor e Intérprete que tiene reconocimiento oficial desde 1980. En ese mismo país, en 1974, el Programa de Traductores de El Colegio de México organizó un programa de posgrado, el primero en la región. En 1986, Cuba creó una escuela para la formación de traductores e intérpretes, consolidando, cuatro años más tarde, en 1972, la Carrera de Traductores e Intérpretes en la Universidad de La Habana, que se caracteriza por combinar el estudio y de la práctica de la profesión desde el inicio de la carrera. En 1970, la Escuela Profesional de Traductores de la Universidad de Puerto Rico habilitó un programa experimental que posteriormente se denominará Programa Graduado en Traducción y que otorga el grado de Magíster. En Colombia, la Carrera de Traductor e Intérprete se creó en el mismo año pero planteada en entidades privadas, como una modalidad del estudio de idiomas extranjeros y con el título de Técnicos Traductores. Al año siguiente, 1971, Brasil creó, en Sao Paulo, la Carrera de Traductor. En este país fue una ley federal, dictada en 1982, la que comenzó a regular los Cursos Superiores de Traductor e Intérprete con el propósito de uniformarlos. También en 1971, la Pontificia Universidad Católica de Chile y la Universidad de Concepción crearon la Carrera de Traductor, con mención en dos idiomas extranjeros y con mención en un idioma extranjero, respectivamente. En 1974, la Universidad Central de Venezuela, a través de la Escuela de Idiomas Modernos, creó la Licenciatura en Traducción e Interpretación. En este mismo año, y por una resolución del Consejo Nacional de la Universidad Peruana, se creó la Carrera de Traducción en dos universidades privadas, la Universidad Ricardo Palma y la Universidad Feminina del Sagrado Corazón.

\section{TRADUCCION Y FORMACION SUPERIOR}

El panorama cronológico recién expuesto muestra una tendencia creciente a situar la formación del traductor en un nivel superior, donde la formación de traductores profesionales idóneos aparece claramente como responsabilidad de la universidad, pues sólo en ella se genera el ámbito apropiado para que los traductores adquieran:

- la formación científica y los conocimientos que garanticen la calidad de su desempeño profesional y

- el sello distinctivo, propio a toda carrera universitaria, que los haga percibir con claridad la importancia de su misión profesional.

En gran parte de estas universidades, conscientes de la importancia de la traducción, se la considera una disciplina, llamada traductología, con problemas propios que deben ser investigados y que requiere para su ejercicio, de profesionales formados a nivel superior, donde la traducción es un excelente punto de partida para una reflexión científica acerca del fenómeno del lenguaje. 
Así concebida, la traducción debe ser objecto de estudio que se aborde científicamente y sólo puede crecer al amparo de la investigación universitaria. Más aún, los centros universitarios en los cuales se lleva a cabo la formación de traductores, enfocadaen un comienzo principalmente como enseñanza de idiomas extranjeros, deberían estar a cargo de docentes investigadores que se nutran con los aportes de la investigación de la comunidad universitaria internacional. Además, por ser un área disciplinaria muy reciente, es en la universidad en donde la traductología podrá crecer y perfeccionarse. Al desarrollarse en la universidad, la actividad traductora compartirá el conocimiento, los métodos y las pautas de rigor de las otras ciencias que se cultivan en los claustros, vale decir, esa común exigencia de análisis profundo y sistemático y esa actitud compartida de crítica rigurosa y de reflexión. Tal convivencia contribuirá necesariamente a que la traductología consolide y acreciente su carácter de disciplina científica. Esta convivencia con otras disciplinas que se cultivan en la universidad no sólo resulta ventajosa para la ciencia de la traducción como área del conocimiento o para la formación de traductores, sino también para otras disciplinas, pues facilmente se verá que la ventaja opera en ambas direcciones.

Para las investigaciones que se desarrollan en campos como el análisis del discurso, la didáctica de la traducción, el análisis del error, los universales lingüísticos, las gramáticas descriptiva y contrastiva, la literatura comparada, la antropología cultural, la sociología, la psicolingüística, la teoría literaria, etc., todo aquel corpus de información validada metódica y rigurosamente por los estudiantes de Traducción y sus profesores, se ha constituido ya en una fuente inapreciable de hipótesis estimulantes que han renovado y enriquecido las perspectivas en una multiplicidad de áreas.

Las traducciones artesanales que realizaban espontáneamente individuos aislados, más o menos dotados o más o menos urgidos por la necesidad de traducir, podán, en casos muy excepcionales, alcanzar excelencia. Sin embargo, está visto que tal modo de operar ya no responde a las demandas que la sociedad contemporánea nos plantea, las que están dadas no sólo por el avance de la ciencia propiamente tal, sino también por la influencia que esta ejerce en el mercado. Los traductores necesitan un armazón sólido que les entregue, por un lado, conocimiento prácticos, un «know-how», y por otro, pautas teóricas que les permitan pensar en su quehacer, enjuiciarlo y perfeccionarlo. Desde un punto de vista académico, no es posible contentarse con practicar con excelencia una actividad, es necesario conocer las causas de lo que se hace. El futuro traductor, docente o investigador en traducción, debe poder llegar a comprender, evaluar y aplicar los muchos y complejos problemas lingüísticos, estilísticos, culturales y psicolinguísticos, entre otros, que se plantean en una traducción. Como dijera Aristóteles, no hay práctica sin teoría: teoría no es más que teoría de la práctica así como la práctica no es otra cosa que praxis de la teoría.

Queda claro, pues, que necesitamos formar traductores que posean una metodología disciplinada y un conocimiento profundo que les permita entregar un producto de una calidad uniformemente alta, acorde con las exigencias crecientes del mercado. Los estándares de rigor y análisis, la perspectiva interdisciplinaria, la vocación de compromiso permanente con la investigación y, sobre todo, el hábito de trabajo solidario y de crítica intersubjectiva son características de un modo de ser netamente universitario.

Postulamos que los contenidos curriculares de un programa universitario de traductores deberían apuntar a un doble objectivo: uno académico y otro profesional. Desde nuestra perspectiva, no es posible contentarse con sólo uno de estos objectivos. Si la orientación curricular fuera más bien academicista, estaríamos ante el eventual peligro de no formar los profesionales que el país requiere. Por el contrario, si la tendencia fuera más bien profesionalizante, podríamos estar descuidando nuestra obligación, como 
universidad, de atender las necesidades de desarrollo científico y tecnológico de un país y de difundir los valores y resultados de la ciencia.

La organización de una Carrera de Traducción que intente cubrir este objectivo académico-profesional, debería tener las siguientes características en lo que se refiere a estructura curricular, perfil del postulante, del egresado y del docente. A nuestro modo de ver, la formación de un traductor debería, además, incorporar elementos que permitieran formar personas con una concepción integral del hombre y de la sociedad, con rigor científico y con una visión cristiana de la vida.

\section{ESTRUCTURA CURRICULAR}

Para lograr este doble objectivo, un programa curricular tendría que contemplar áreas en forma interdependiente, de modo que el diseño curricular resultara integrador. Las áreas deberían apuntar a :

- una orientación teórica que permitiera al estudiante abordar creativamente tareas de análisis y desarrollo respecto al proceso de traducción y a áreas disciplinarias afines, a la vez que reflexionar críticamente sobre su trabajo. Los alumnos deberían ser capaces de seleccionar, diferenciar, contrastar y evaluar los elementos y las etapas propias de un proceso de traducción tanto desde una perspectiva bilingüe o multilingüe, así como sugerir y realizar proyectos de investigación en su especialidad;

- una orientación tecnológico-aplicada que permitiera al estudiante adquirir criterios, destrezas y habilidades que garantizaran un desempeño profesional riguroso como traductor bilingüe o multilingüe, al español. Los alumnos deberían ser capaces de dominar, aplicar, criticar y evaluar las técnicas y los métodos del proceso de la traducción así como los productos del mismo y también las herramientas computacionales que apoyen su labor.

En general, el curriculum tendría que ofrecer a los alumnos un conjunto coherente de estudios en traductología: una sólida formación en lingüística y en epistemología, teoría y práctica de la traducción, a la vez que la posibilidad de especializarse o de complementar sus estudios con un conjunto de cursos electivos en diferentes áreas.

Partiendo de estos postulados, la Carrera de Traducción de la Pontificia Universidad Católica de Chile ha reformulado su curriculum. Este contempla, por un lado, cursos teóricos sobre Traductología (dos niveles), Lingüistica del Texto, Filología Románica, Semiótica, Metodología de la Investigación en Traducción, Terminlogía, Semántica y Lexicología. Y por el otro, asignaturas en las que se persigue sólo un cierto grado de especialización, como las materias de Traducción Técnica y Científica, Seminarios de Traducción Literaria, Evaluación de traducciones, Problemas de la traducción y Tecnolectos. Debido a que el diploma que otorga la Pontificia Universidad Católica de Chile es de Traductor desde dos idiomas extranjeros al español, el programa incluye un porcentaje muy importante de cursos sobre Gramática y Lingüística españolas, Talleres de redacción y cursos de Corrección de estilo, en español. Finalmente, en materia de cursos electivos, el alumno podrá optar entre las diversas asignaturas que ofrezca la universidad : Lógica, Introducción al Derecho, a la Economía, cursos de Ciencia Política, Latín, Griego, cursos en áreas científicas, etc.

Un requisito fundamental de titulación para un traductor formado a nivel superior es la realización de una práctica profesional (stage) ya que es a través de ella que el alumno se forma una visión general, objectiva y concreta de lo que será su trabajo profesional futuro en el mercado laboral. En esta etapa, el alumno deberá estar en condiciones, en nuestro caso, de traducir al español, en forma rigurosa y científica, textos técnicos, científicos y literarios de los idiomas extranjeros estudiados, y además, estar capacitado para el análisis y la investigación de problemas especificos que surgen en el proceso de la traducción. 
La realización de la práctica profesional (320 horas de trabajo práctico en cada idioma) en empresas o institiciones públicas o privadas cumple el doble propósito de:

- proveer a los alumnos de un lugar de trabajo donde el practicante pueda aquilatar y poner a prueba su formación profesional, sus conocimientos y su experiencia como universitario, conjuntamente con sus características personales;

- crear e impulsar la necesidad por la profesión o el cargo de traductor, aspecto que es fundamental para consolidar el nivel de la profesión. En este punto, es muy interesnte comentar la situación de Chile, donde ha habido literalmente un vuelco en el ambiente laboral del traductor, producido por la «invasión» de traductores universitarios que han egresado de nuestras universidades y también por la creciente actividad económica de Chile. Así, podemos comprobar con orgullo que numerosas empresas han creado puestos de traductor especialmente para contratar a nuestros egresados.

Debido a este cambio notorio en el mercado, el Departamento de Traducción de nuestra universidad decidió crear, en 1986, un Servicio de Traducciones. Este servicio ha venido a llevar un vacío en el mercado, exigente en cuanto a calidad y ha permitido dar empleo a nuestro mejores egresados.

\section{PERFIL DEL POSTULANTE Y DEL EGRESADO}

Los postulantes a una Carrera de Traducción deberían poseer competencia en lenguas extranjeras, por lo menos en un nivel intermedio, ya que no corresponde a la universidad iniciarlos en el estudio de las mismas. En lo que se refiere a combinación de idiomas extranjeros, se deberá tender idealement a una fórmula lingüística de dos idiomas. De lo contrario, el mercado se vería reducido inevitablemente desde un comienzo.

La realidad actual en Chile, y todo hace pensar que la situación no es radicalmente diferente en otros países hispanohablantes, nos lleva a sugerir que cualquier combinación debería incluir el idioma inglés. Incluso es aconsejable que, como parte de sus materias electivas, los alumnos sigan cursos de traducción hacia el inglés, el alemán o hacia el francés, pues es corriente que en su vida profesional se vean enfrentados a tal tipo de requerimientos.

Los currícula podrán incluir cursos de enseñanza de idioma extranjero sólo en niveles avanzados. Por un lado, no hay que substraerse a la realidad de nuestros países latinoamericanos en que las escuelas secundarias no entregan graduados con el nivel de competencia linguística que un programa de traducción exige. Por otro, tampoco es posible que un aspirante a Traducción debe aprender en la universidad, las lenguas de las cuales desea traducir. Más bien, lo que deberá procurarse, en el ámbito lingüístico, es una enseñanza de tipo complementaria a las habilidades que requiere un traductor: comprensión a cabalidad de las lenguas extranjeras, estrategias de quiebre linguiístico, interpretación y análisis de textos, aproximación a la lectura, etc.

Con respecto a otras características necesarias en los postulantes, estimamos que deberían aplicarse pruebas que midieran la inteligencia verbal (capacidad de análisis y síntesis, entre otros), competencia en el manejo de la lengua materna, aptitud específica para traducir (ejercicios de traducción) y nivel cultural.

En relación con el egresado, concurren dos tipos de componentes que, aunque muy relacionados, conviene distinguir. Por un lado, tenemos los que llamaremos de adquisición de conocimientos y por otro, que son igualmente importantes los que llamaremos de formación de habilidades y criterios.

Dentro del primero de estos grupos se pretende que el traductor universitario:

a) domine el repertorio de formas y significaciones de las lenguas con las que opera, percibiendo e identificando en los textos, diversas funciones y registros por sofisticados que ellos sean; 
b) conozca la variedad de códigos y estilos culturales que coexisten y que determinan los patrones de comunicación dentro y entre cada grupo y sub-grupo de hablantes y

c) conozca una metodología de investigación que le permita organizarse y generar estrategias eficaces para el análisis, el estudio, la evaluación y la resolución de los problemas que encuentre en el proceso de traducción.

Dentro del segundo grupo, habilidades y criterios, señalaremos como objectivos de esta formación:

a) adquirir la práctica para discriminar con finura y para evaluar certeramente, y en textos muy diversos, todas las funciones específicas que cumplen cada una de las elecciones de los autores de los textos originales, paralelamente, fundamentar y justificar en sus propios textos traducidos las opiniones correspondientes a cada una de ellas;

b) adquirir una sagacidad especial para percibir el sentido profundo y global de los mensajes y así poder buscar los formatos de organización retórica adecuada que los reflejen, evitando introducir distorsiones que malogren o desvirtúen la intención de los originales, o violenten las formas de la lengua a la cual traducen. Paciencia, prolijidad, sensibilidad, respecto por la lengua y por la interpretación correcta de la intención de los mensajes son, todas ellas, cualidades que los traductores deben ir adquiriendo progresivamente;

c) recurrir a un repertorio amplio de obras de consultas que le permitan recurrir a las fuentes de referencia apropiadas - orales y/o escritas - tales como diccionarios, glosarios, gramáticas y documentación en general;

d) recurrir a toda aquellas herramientas computacionales que puedan facilitar y hacer más eficiente su labor como traductor;

e) adquirir criterios que, frente a una tarea concreta, les permittan cuanta atención, tiempo y dedicación se requiera. Debe, por tanto, poder barajar responsablemente factores, entre otros, como la urgencia con que el texto se necesita, la naturaleza propia de este y los destinatarios que de él se servirán y

f) desarrollar sanos criterios de autocrítica que, en determinados casos le permitan reconocer sus propias limitaciones y que lo lleven, sin inhibiciones, a buscar el apoyo de colegas más especializados o el de informantes expertos que puedan ayudarlo a cumplir su cometido sin que por ello se sienta vulnerado en su dignidad profesional.

\section{PERFIL DEL DOCENTE FORMADOR DE TRADUCTORES}

Hacemos énfasis en la necesidad de una elección cuidadosa del cuerpo docente formador de traductores. Idealmente, los cursos de traducción propiamente tales deberían estar a cargo de traductores profesionales en ejercicio, con formación universitaria, los que tendrían que complementar, permanentemente, sus conocimientos con nociones de pedagogía universitaria. Es decir, con todos aquellos aspectos que harán del proceso de enseñanza-aprendizaje algo efectivo: nociones de evaluación, de preparación y selección de material docente, de metodología de la investigación, de didáctica de la traducción, etc.

De acuerdo con Jean Delisle, un pedagogo de la traducción debe:

- conocer la naturaleza exacta de la operación traductora, ser buen traductor y haber reflexionado acerca de los mecanismos de transferencia de sentido de una lengua extranjera a la materna;

- saber relacionar práctica y teoría;

- ser capaz de aislar las dificultades de traducción para luego jerar quizarlas y facilitar, de esta manera, la adquisición de las técnicas de traducción por parte de los alumnos;

- organizar la enseñanza de acuerdo con objetivos de aprendizaje muy bien definidos; 
ser capaz de hacer descubrir al alumno las facetas del manejo de la lengua desde una perspectiva de comunicación interlingüística;

- saber transmitir su experiencia práctica y

- alimentar la reflexión teórica, con la observación de los aciertos y los errores de sus alumnos ${ }^{1}$.

Según nuestro parecer, los egresados de un plan de estudios así concebido podrán enfrentar idóneamente las exigencias del mercado. Cuando hablamos de mercado, no nos estamos refiriendo solamente al mercado tradicional, es decir, a las empresas o institutiones del sector público y privado que contratan traductores para llenar su propia necesidad de traducción. Hemos ampliado el concepto de mercado, incluyendo en él a las instituciones de educación superior formadoras de traductores que requieren, en forma imperiosa, contar con los mejores elementos docentes e investigadores que, naturalmente, provendrán de sus mejores egresados.

(Ponencia presentada en el «Primer Congreso Argentino de Traductores e Intérpretes», Universidad de Buenos Aires, Buenos Aires, Argentina, septiembre de 1987.)

NOTE :

1. DELISLE, J. (1981): L'enseignement de l'interprétation et de la traduction, de la théorie à la pédagogie, Éditions de 1'Université d'Ottawa, Ottawa, Canada. Esta cita la hemos traducido exclusivamente para los efectos del presente trabajo.

BIBLIOGRAPHIE :

CABRERA, I. ET AL. (1984): Estudio del campo ocupacional del traductor en Santiago de Chile, Facultad de Letras, Santiago de Chile, Pontificia Universidad Católica de Chile.

DELISLE, J. (1981): L'enseignement de l'interprétation et de la traduction, de la théorie à la pédagogie, Ottawa, Éditions de l'Université d'Ottawa.

GARCIA YEBRA, V. (1983): Teoría y Práctica de la traducción, 2 vol., Madrid, Ed. Gredos.

HÖRMANN, P. (1988): «La traducción como agente de desarrollo», ponencia presentada en el Primer Simposio Latinoamericano de Terminología, abril, Caracas, Universidad Simón Bolívar, (en prensa).

HÖRMANN, P., MIZON, M.I., TROSKO, B., VON MOLTKE, R. (1986) : «Proyecto de creación del Ciclo Básico y Ciclo Terminal en Letras con mención en Traducción», Documento Interno de Trabajo, Departamento de Traducción, Instituto de Letras, Santiago de Chile, Pontificia Universidad Católica de Chile.

RONDEAU, G. (1984) : Introduction à la terminologie, Québec, Gaëtan Morin. 